\title{
Plant infection and the establishment of fungal biotrophy
}

\author{
Kurt Mendgen and Matthias Hahn
}

\begin{abstract}
To exploit plants as living substrates, biotrophic fungi have evolved remarkable variations of their tubular cells, the hyphae. They form infection structures such as appressoria, penetration hyphae and infection hyphae to invade the plant with minimal damage to host cells. To establish compatibility with the host, controlled secretory activity and distinct interface layers appear to be essential. Colletotrichum species switch from initial biotrophic to necrotrophic growth and are amenable to mutant analysis and molecular studies. Obligate biotrophic rust fungi can form the most specialized hypha: the haustorium. Gene expression and immunocytological studies with rust fungi support the idea that the haustorium is a transfer apparatus for the long-term absorption of host nutrients.
\end{abstract}

DOI: 10.1016/S1360-1385(02)02297-5

Kurt Mendgen

Dept of Biology, Phytopathology,

University of Konstanz,

Universitätsstrasse 10,

78457 Konstanz,

Germany.

e-mail: kurt.w.mendgen@

uni-konstanz.de

Matthias Hahn

Dept Biology, University of Kaiserslautern,

Post Box 3049, 67653

Kaiserslautern, Germany.
As early as 1866, the German botanist Anton De Bary observed that plant parasitic fungi alter the morphology of their hyphae in response to structural and physiological features of the host surface soon after germination [1]. They form infection structures that accomplish crucial stages of pathogenesis, including attachment, host recognition, penetration, proliferation and nutrition. Infection structure formation is controlled by complex regulatory pathways and accompanied by regulated gene expression [2].

After host invasion, fungi use different strategies to gain access to host nutrients. Whereas necrotrophs (e.g. the corn leaf blight fungus Cochliobolus heterostrophus) quickly kill plant cells to feed subsequently as saprotrophs, other fungi maintain biotrophic relationships with their hosts either transiently or until sporulation. The biotrophic lifestyle is realized in a remarkable range of ways: intercellular (Cladosporium fulvum); subcuticular (Venturia inaequalis); inter- and intracellular (Claviceps purpurea, Ustilago maydis, monokaryotic rust fungi); extracellular with haustoria within epidermal cells (powdery mildews); intercellular with haustoria within parenchyma cells (dikaryotic rust fungi and downy mildews). A transient type of biotrophy is observed in the so-called hemibiotrophic fungi (Magnaporthe grisea, Phytophthora infestans and Colletotrichum spp.).

Our understanding of biotrophy is still limited. With few exceptions, the most important, haustorium-forming biotrophs cannot be grown in artificial culture, and no useful transformation systems are available. By contrast, C. purpurea, $U$. maydis and several hemibiotrophs are well studied and amenable to modern molecular tools.
Molecular studies of obligate and non-obligate biotrophs are beginning to provide insights into the nature of biotrophy.

To our knowledge, the following properties appear to be hallmarks of biotrophic fungi: (1) highly developed infection structures; (2) limited secretory activity, especially of lytic enzymes; (3) carbohydraterich and protein-containing interfacial layers that separate fungal and plant plasma membranes; (4) long-term suppression of host defense;

(5) haustoria, which are specialized hyphae for nutrient absorption and metabolism. In this article, we discuss recent data about cytological and molecular aspects of fungal biotrophy. To keep within the available space, we restrict our focus to Colletotrichum spp. and rust fungi, which show different degrees of biotrophic growth.

The bean pathogen Colletotrichum lindemuthianum (Fig. 1) is the classical example of a hemibiotrophic fungus. After penetrating the host cuticle and the epidermal cell wall, it initially grows as a biotroph with primary intracellular hyphae for one or a few days. Subsequently, secondary, narrower hyphae are formed that kill the host cells and proliferate by necrotrophic growth [3]. The rust fungi (Fig. 2), by contrast, are obligately biotrophic, haustorium-forming plant pathogens that can establish stable interactions with their hosts that can last for months $[4,5]$.

The early stages of infection (adhesion, appressorium formation and penetration) have been reviewed in detail recently for several plant pathogenic fungi including Colletotrichum and rust fungi $[6,7]$. They are not fundamentally different for fungi with contrasting nutritional properties. Their purpose is to ensure reliable infection while causing minimal damage to the host cells. In M. grisea and probably also in Colletotrichum spp., melanin impregnation of the appressorium wall enables them to build up an extraordinarily high osmotic pressure. This is translated into a penetration force of $16.8 \pm 3.2 \mu \mathrm{N}$, concentrated at the tip of a thin penetration hypha (penetration peg) of Colletotrichum graminicola [8]. The most sensitive mode of surface recognition is found in the rust fungi. The tip of their dikaryotic germ tube can follow topographical features of the plant cuticle, which increases the probability of encountering stomatal openings. Uromyces appendiculatus recognizes the lip height of the bean guard cell $(0.5 \mu \mathrm{m})$ [9]. Chemical signals, such 


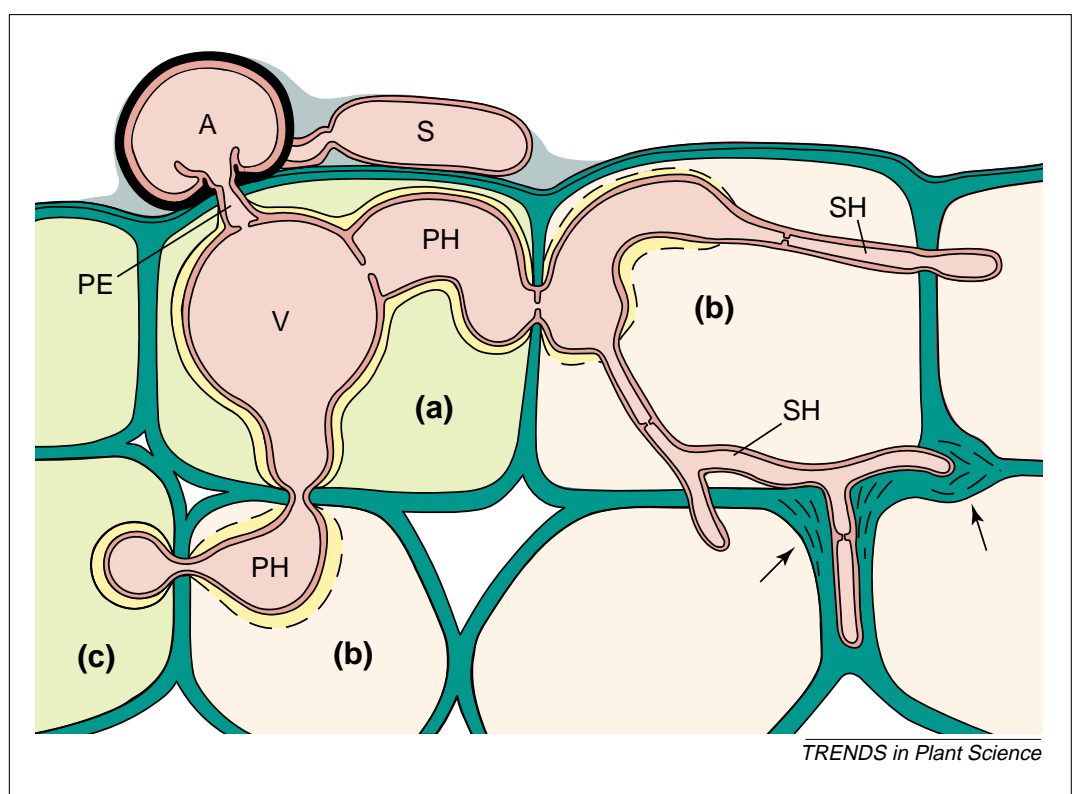

Fig. 1. Hemibiotrophic infection by Colletotrichum lindemuthianum. A spore (S) attached to the host surface germinates to form a short germ tube, which differentiates into a domed, melanized appressorium (A). The penetration hypha (PE) develops on the appressorium base, transforming internal pressure into mechanical force to pierce the cuticle and the cell wall. The penetration hypha swells within the epidermal cell to form a vesicle (V) and broad primary hyphae (PH), which are surrounded by the invaginated plant plasma membrane. The host protoplast remains alive during the biotrophic stage (a) and an interfacial matrix separates the protoplasts of fungus and host (yellow). One or two days after penetration, plant plasma membrane disintegration starts, leading to host cell death (b). As new host cells are colonized by primary hyphae, the sequence of a transient biotrophic phase followed by cell killing is repeated (c). This relationship ends as soon as narrow secondary hyphae (SH) develop, which are not surrounded by the host membrane and lack an interfacial matrix. Host walls break down because of the secretion of large amounts of cell-wall-degrading enzymes by the secondary hyphae (arrows).

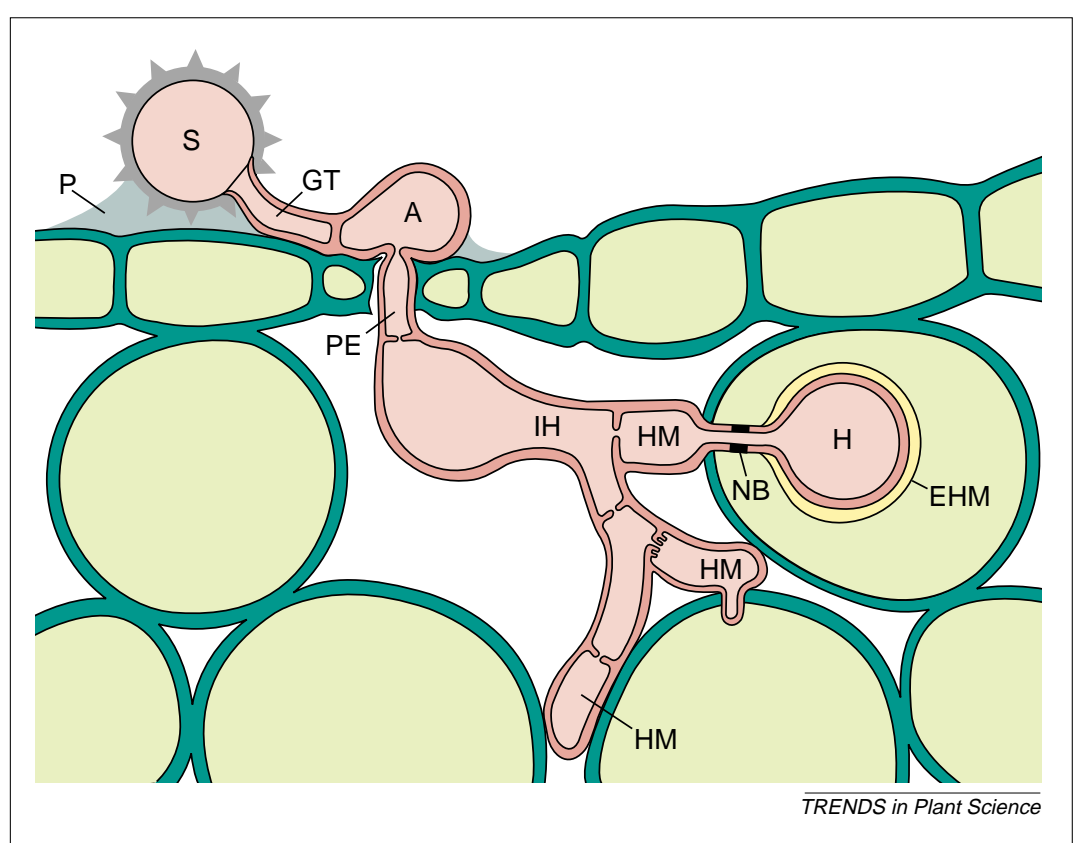

Fig. 2. Biotrophic infection by Uromyces fabae (dikaryon). A germ tube (GT) emerges from an urediospore $(\mathrm{S})$ attached to the host by an adhesion pad (P). After recognition of the guard cell lip, an appressorium (A) develops over the stomatal pore. The penetration hypha (PE) penetrates into the substomatal chamber and elongates into an infection hypha $(\mathrm{IH})$. When the tip of the infection hypha contacts a host cell wall, a haustorial mother cell $(\mathrm{HM})$ is formed from which the haustorium $(\mathrm{H})$ invades the host cell. Unique features of the dikaryotic haustorium are the dark-staining neckband (NB) around the haustorial neck and the interfacial, extrahaustorial matrix (yellow) surrounded by the extrahaustorial membrane (EHM). After forming the first haustorium, the infection hypha branches and further intercellular hyphae, haustorial mother cells (HM) and haustoria are formed. as leaf alcohols can contribute to this unique recognition system [10].

Work with artificially induced infection structures from Uromyces fabae revealed that secretion of cell-wall-degrading enzymes is under strict developmental control. They were found not to be secreted until appressoria and infection hyphae (cellulases, pectin esterases) or haustorial mother cells (pectate lyases) were formed [11]. It was suggested that the order and site of their appearance correlate with the demand for highly localized host-cell penetration during haustorium formation within the leaf.

\section{Hemibiotrophic development}

A range of infection strategies is found in the large genus Colletotrichum. Most species (e.g. C. lindemuthianum and Colletotrichum destructivum) are hemibiotrophs, but others, (e.g. Colletotrichum capsici) display 'subcuticular, intramural necrotrophy' [3], which is to say that they initially grow within the cell walls of host epidermal cells before they proliferate rapidly through the tissue that has been killed. Colletotrichum gloeosporioides follows both strategies, depending on the host plant [12]. In addition, several Colletotrichum spp. are known to form long-term quiescent infections [13,14].

Cytological pictures of (hemi)biotrophic interfaces using ultrarapid freezing followed by freeze substitution are available mainly for C. lindemuthianum. After penetration, the intracellular infection vesicle and the primary hyphae colonize only a few host cells. Both are surrounded by a matrix that separates the fungal cell wall from the invaginated host plasma membrane (Fig. 3a). This matrix is extracytoplasmic and connected to the plant apoplast. Within the interfacial matrix, a fungal glycoprotein, encoded by CIH1, was identified [15]. The protein was shown to be present uniquely at this interface in the biotrophic stage of hemibiotrophic Colletotrichum spp. Its expression was switched off at the onset of necrotrophic development [16].

Insertional mutagenesis has been used with remarkable success to identify genes that are involved in parasitic development of Colletotrichum. In $C$. gloeosporioides, screening for nitrogenstarvation-induced genes resulted in the cloning of CgDN3. A mutant disrupted in this gene provoked a hypersensitive-like response on intact leaves of the host plant, Stylosanthes guianensis. CgDN3 is specifically expressed during early biotrophic growth [17] and might be required to maintain the initial stage of intracellular development

In C. lindemuthianum, a nonpathogenic mutant obtained by random mutagenesis was still able to form biotrophic primary hyphae, but no secondary hyphae were observed. It was suggested that the mutant was blocked in the switch from biotrophic to necrotrophic growth. Evidence that the mutant has a regulatory nature was supported by sequencing of the 


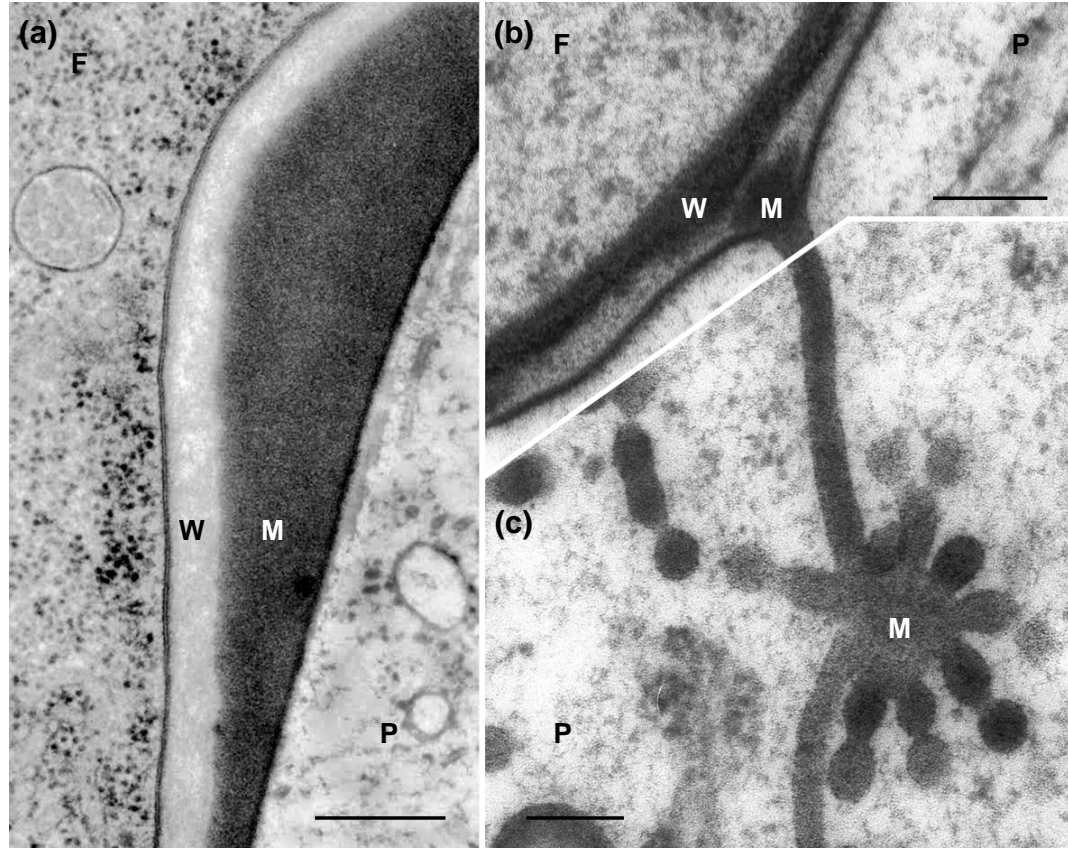

Fig. 3. Comparison of the biotrophic interfaces in host plant leaves infected by Colletotrichum lindemuthianum or Puccinia hemerocallidinis. These samples were prepared by high-pressure freezing and freeze substitution. Scale bars $=250 \mathrm{~nm}$. (a) In bean leaves infected by $C$. lindemuthianum, the fungal cytoplasm (F) with the plasma membrane is separated by a fungal wall (W) and a fine granular interfacial matrix $(M)$ from the plant cytoplasm $(P)$ with the invaginated but unspecialized host plasma membrane (photograph courtesy of Richard J. O'Connell and Kurt Mendgen). (b) In day-lily leaves infected by $P$. hemerocallidinis, the cytoplasm of the fungal haustorium $(F)$ is surrounded by the haustorial wall $(\mathrm{W})$ and the extrahaustorial matrix $(\mathrm{M})$. The extrahaustorial membrane of the plant follows the contours of the extrahaustorial matrix and is continuous with tubular elements extending into the cytoplasm of the host plant (P). (c) Tubular elements continuous with the extrahaustorial matrix (M) reach far into the host cytoplasm (P) (photograph courtesy of Charles W. Mims, Carmen Rodriguez-Lother and Elizabeth A. Richardson).

disrupted gene, CLTA1, which appears to be a transcriptional activator belonging to the zinc cluster family [18].

In the cucurbit pathogen Colletotrichum magna, a large-scale screening of REMI mutants resulted in many isolates that caused symptomless infections [13]. Interestingly, these mutants conferred on the infected host plant almost complete disease protection against wild-type C. magna and other fungal pathogens [19]. Apparently, mutations in several genes can modify a fine balance between non-destructive host colonization and pathogenesis. It will be interesting to understand more precisely the role of these genes in the infection process of $C$. magna and their occurrence in other hemibiotrophic fungi.

After completion of the biotrophic phase, narrow necrotrophic hyphae develop [20] and start to secrete large amounts of cell-wall-macerating enzymes such as endopolygalacturonases [21]. In C. gloeosporioides, a pelB-encoded pectate lyase was found to be an important virulence factor in avocado fruit. The enzyme not only degrades cell-walls but also reduces host defense reactions that are induced by the release of oligogalacturonides [22]. Some Colletotrichum spp. produce ammonia in response to acidic $\mathrm{pH}$, which seems to increase the $\mathrm{pH}$ in vitro and in the host plant to the optimum level for pectate lyase activity [23].
In C. graminicola, an insertion mutant showing a reduction in transcript levels of a gene $(C P R 1)$ encoding a putative signal peptidase resulted in a mutant that was defective in pathogenesis. Cytological studies indicated that the mutant continued to grow with primary hyphae to a limited extent but was unable to switch to necrotrophic growth. It was speculated that the mutant's enzyme secretion was affected [24].

\section{Biotrophic development}

Intercellular hyphae of rust fungi (dikaryon) begin host-cell penetration of the leaf from haustorial mother cells. They are reminiscent of appressoria in that they also have a thick, multilayered wall that attaches firmly to the host wall and forms a penetration hypha to invade the host cell [25-27]. Whether the functional similarity of appressoria and haustorial mother cells is echoed on a molecular level remains to be shown. One or more signals of the host are needed to complete the differentiation of the haustorium [28]. For this, the penetration hypha develops into the haustorial neck and enlarges to produce the haustorial body. The whole structure, although reaching far into the host cell, remains surrounded by a modified host plasmalemma, the extrahaustorial membrane [26]. In contrast to intercellular hyphae, which tend to become vacuolated, fungal cytoplasm first accumulates within haustorial mother cells and then migrates into haustoria [29]. This cytological picture suggests that major metabolic activities occur within haustoria, and this is supported by molecular studies.

From a haustorium-specific cDNA library of the broad bean rust $U$. fabae, in planta-induced genes were isolated that are induced in the biotrophic mycelium. Of these, two thiamine-biosynthesis genes (THI1 and THI2) were found to be most highly expressed in haustoria, together representing $\sim 5 \%$ of the haustorial mRNA [30]. Immunocytology revealed a preferred location of the THI1-encoded protein in the haustorial cytoplasm. Thiamine diphosphate (vitamin $\mathrm{B}_{1}$ ) is a cofactor of several enzymes of amino acid and carbon metabolism. These data suggest that haustoria play a central role in primary metabolism.

Gene expression and immunolocalization data also support the long-held idea [31] that rust haustoria are specialized for the uptake of host assimilates. Another gene (HXT1) that is abundantly expressed in rust haustoria but barely transcribed in other fungal structures encodes a hexose transporter [32]. Functional expression in Saccharomyces cerevisiae and Xenopus laevis oocytes revealed a substrate specificity of the $H X T 1$-encoded protein towards the hexoses glucose and fructose, and demonstrated a proton-driven symport mechanism. Immunocytochemical analysis of HXT1p suggests that the fungus concentrates this transporter in the haustorial plasma membrane (Fig. 4). Furthermore, genes encoding three (putative) amino acid 


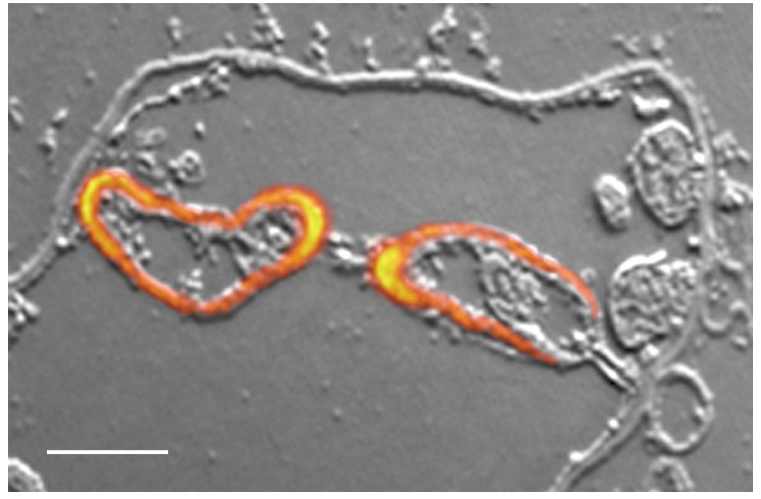

Fig. 4. Localization by immunofluorescence of the hexose transporter HXT1p in the periphery of two mature haustoria. Proximal parts of the haustorial body and the haustorial neck are not labeled. This image was constructed by superimposing $\mathrm{Cy} 3$ fluorescence and differential interference contrast images. Scale bar $=5 \mu \mathrm{m}$.

transporters have been characterized. $A A T 1$ transcripts are present in germinated hyphae and throughout parasitic hyphae but accumulate to the highest levels in haustoria [33].AAT1p is a proton symporter with highest activities for histidine and lysine [34]. The AAT2-encoded protein shows high similarity to AAT1p (55.1\% identity) and was detected by immunofluorescence in haustoria only [35]. AAT3 was found to encode a low-specificity amino-acid-proton symporter with a preference for S-containing amino acids (C. Struck et al., unpublished).

These studies show that the bulk of sugars and amino acids are taken up by haustoria, although they represent only a minor volume and surface fraction of the total biotrophic mycelium. According to our model, an $\mathrm{H}^{+}$-ATPase generates the proton gradient across the haustorial plasma membrane, which provides the energy for the uptake of nutrients from the extrahaustorial matrix [36,37]. How these nutrients are translocated from the infected host cell across the extrahaustorial membrane is unknown.

The efficiency of haustorial nutrient uptake is probably increased by the so-called neckband in the area of the haustorial neck, a structure comparable to the Casparian strip in roots [38]. It bridges fungal and host plasma membranes and thus seals the plant apoplast from a newly generated interfacial apoplast around the haustorial body. This extrahaustorial compartment consists of a thin fungal wall with new carbohydrate components [39] and a prominent extrahaustorial matrix containing plant-cell-wallrelated carbohydrates and enzymes such as $\beta$-1,3-glucanases $[40,41]$. It seems that the extrahaustorial interface represents the 'trade center' for the inter-organismic transfer of signals and nutrients. The fungus probably contributes to it by secreting cytokinins [42], invertase [43] and other proteins that might all regulate the outcome of the infection. The extrahaustorial membrane also displays unusual features that are distinct from those of the plant plasma membrane from which it is derived: in electron-microscope pictures, it lacks intramembrane particles, has a reduced content of glycolipids [12] and forms tubular extensions that are sometimes filled with dark-staining material. In the case of lily leaves infected by Puccinia hemerocallidis [44], these extensions produce a tubular network that penetrates deep into the host cytoplasm (Fig. 3b,c). Obviously, the extrahaustorial membrane is a unique type of parasite-induced plant membrane.

\section{Conclusion}

The common goal of biotrophic fungi (to invade host tissue with minimal damage of plant cells) is accomplished in a range of ways. Early infection structures do not appear to be fundamentally different from those of necrotrophic fungi but they generally show more functional specialization, such as the ability to recognize host-specific surface features (e.g. the size of stomatal lips in the case of rust fungi). Within the host tissue, biotrophic hyphae do not provoke host cell death. How this is achieved is still far from being understood but an important factor is certainly a strict limitation of the secretion of cell-wall-degrading enzymes. Nevertheless, secretory activity is required by both partners to form the interface layers that are observed in biotrophic interactions. They contribute in an unknown way to the maintenance of compatibility and to the lack of host defense mechanisms [35,45].

Nonpathogenic mutants of Colletotrichum spp. have proved to be a rich source of genes that seem to be involved in the transition from biotrophy to necrotrophy. Other fungi, such as $M$. grisea and $U$. maydis, are also important models for an understanding of various aspects of biotrophic parasitism and already provide genomic approaches. However, the different strategies adopted by biotrophic fungi cannot be explained by a few model systems $[46,47]$. For instance, structural and functional properties of haustoria need to be studied in those fungi that produce them.

There are many open questions for future research. For instance, how does the fungus manipulate the physiology of the host? What are the borderlines between parasitic and mutualistic associations, including those with endophytic fungi [48]? From the host side, we need to know much more about the contributions of the plant to the development and the outcome of the interaction. A screen for host mutants that do not support the growth of biotrophic pathogens has been performed in Arabidopsis [49]. In this respect, the legume Medicago truncatula is emerging as another model plant that forms interactions with arbuscular mycorrhiza, rust and Colletotrichum spp. [50].

For the next few years, the coordinated application of molecular and microscopic tools [51-53] and the exploitation of functional genomics and proteomics promise to give us exciting insights into the mechanisms of the intimate interactions between fungi and plants. 


\section{References}

1 De Bary, A. (1866) Morphologie und Physiologie der Pilze, Flechten und Myxomyceten, Wilhelm Engelmann, Leipzig, Kingdom of Saxony

2 Kahmann, R. and Basse, C. (2001) Fungal gene expression during pathogenesis related development and host plant colonization Curr. Opin. Microbiol. 4, 374-380

3 O'Connell, R.J. et al. (2000) Dissecting the cell biology of Colletotrichum infection processes. In Colletotrichum: Host specificity, Pathology, and Host-Pathogen Interaction (Prusky, D. et al., eds), pp. 57-77, APS Press

4 Mendgen, K. et al. (2000) Biotrophy and rust haustoria. Physiol. Mol. Plant Pathol. 56, 141-145

5 Staples, R.C. (2000) Research on the rust fungi during the twentieth century. Annu. Rev. Phytopathol. 38, 49-69

6 Tucker, S.L. and Talbot, N.J. (2001) Surface attachment and pre-penetration stage development by plant pathogenic fungi. Annu. Rev. Phytopathol. 39, 385-417

7 Hardham, A.J. (2001) Cell biology of fungal infection of plants. In The Mycota: Biology of the Fungal Cell (Vol. VIII) (Howard, R.J. and Gow, N.A.R., eds), pp. 91-124, Springer-Verlag

8 Bechinger, C. et al. (1999) Optical measurements of invasive forces exerted by appressoria of a plant pathogenic fungus. Science 285, 1896-1899

9 Staples, R.C. and Hoch, H.C. (1997) Physical and chemical cues for spore germination and appressorium formation by fungal pathogens. In The Mycota: Plant Relationships (PartA, Vol. VI) (Carroll, G. and Tudzynski, P., eds), pp. 27-40, Springer Verlag

10 Collins, T.J. et al. (2001) Synergistic induction of wheat stem rust appressoria by chemical and topographical signals. Physiol. Mol. Plant Pathol. $58,259-266$

11 Deising, H. et al. (1995) Differentiation and cell wall degrading enzymes in the obligately biotrophic rust fungus Uromyces viciae-fabae. Can. J. Bot. 73 (Suppl. 1), 624-631

12 Perfect, S.E. and Green, J.R. (2001) Infection structures of biotrophic and hemibiotrophic fungal plant pathogens. Mol. Plant Pathol. 2, 101-108

13 Redman, R.S. et al. (1999) Conversion of the pathogenic fungus Colletotrichum magna to a nonpathogenic, endophytic mutualist by gene disruption. Mol. Plant-Microbe Interact. 12, 969-975

14 Beno-Moualem, D. and Prusky, D. (2000) Early events during quiescent infection development by Colletotrichum gloeosporioides in unripe avocado fruits. Phytopathology 90, 553-559

15 Perfect, S.E. et al. (1998) Expression cloning of a fungal proline-rich glycoprotein specific to the biotrophic interface formed in the Colletotrichum-bean interaction. Plant J. 15, 273-279

16 Perfect, S. et al. (2000) The distribution and expression of a biotrophy-related gene, $\mathrm{CIH1}$ within the genus Colletotrichum. Mol. Plant Pathol. 1, 213-221

17 Stephenson, S-A. et al. (2000) CgDN3: an essential pathogenicity gene of Colletotrichum gloeosporioides necessary to avert a hypersensitive-like response in the host Stylosanthes guianensis. Mol. Plant-Microbe Interact. 13, 929-941
18 Dufresne, M. et al. (2000) A GAL4-like protein is involved in the switch between biotrophic and necrotrophic phases of the infection process of Colletotrichum lindemuthianum on common bean. Plant Cell 12, 1579-1589

19 Redman, R.S. et al. (1999) Biochemical analysis of plant protection afforded by a nonpathogenic endophytic mutant of Colletotrichum magna. Plant Physiol. 119, 795-804

20 Perfect, S.E. et al. (2001) Surface characteristics of necrotrophic secondary hyphae produced by the bean anthracnose fungus, Colletotrichum lindemuthianum. Eur. J. Plant Pathol. 107, 813-819

21 Centis, S. et al. (1997) Endopolygalacturonase genes from Colletotrichum lindemuthianum: cloning of CLPG2 and comparison of its expression to that of $C L P G 1$ during saprophytic and parasitic growth of the fungus Mol. Plant-Microbe Interact. 10, 769-775

22 Yakoby, N. et al. (2001) Colletotrichum gloeosporioides pelB is an important virulence factor in avocado fruit-fungus interaction. Mol. Plant-Microbe Interact. 14, 988-995

23 Prusky, D. et al. (2001) Local modulation of host $\mathrm{pH}$ by Colletotrichum species as a mechanism to increase virulence. Mol. Plant-Microbe Interact. $14,1105-1113$

24 Thon, M.R. et al. (2002) CPR1: a gene encoding a putative signal peptidase that functions in pathogenecity of Colletotrichum graminicola. Mol. Plant-Microbe Interact. 15, 120-128

25 Harding, M.W. et al. (1999) Host-parasite relationships in bean cultivars of varying susceptibility to bean rust. Can. J. Bot. $77,1551-1559$

26 Heath, M.C. (1997) Signalling between pathogenic rust fungi and resistant or susceptible host plants. Ann. Bot. 80, 713-720

27 Zhang, L. and Dickinson, M. (2001) Fluorescence from rust fungi: a simple and effective method to monitor the dynamics of fungal growth in planta. Physiol. Mol. Plant Pathol. 59, 137-141

28 Heath, M.C. (1990) Influence of carbohydrates on the induction of haustoria of the cowpea rust fungus in vitro. Exp. Mycol. 14, 84-88

29 Mims, C.W. et al. (2001) Ultrastructure of the host-parasite interaction in leaves of Duchesnea indica infected by the rust fungus Frommeela mexicana var. indicae as revealed by high pressure freezing. Can. J. Bot. 79, 49-57

30 Sohn, J. et al. (2000) High level activation of vitamin $B_{1}$ biosynthesis genes in haustoria of the rust fungus Uromyces fabae. Mol. Plant-Microbe Interact. 13, 629-636

31 Staples, R.C. (2001) Nutrients for a rust fungus: the role of haustoria. Trends Plant Sci. 6, 496-498

32 Voegele, R.T. et al. (2001) The role of haustoria in sugar supply during infection of Vicia faba by the rust fungus Uromyces fabae. Proc. Natl. Acad. Sci. U. S. A. 98, 8133-8138

33 Wirsel, G.R. et al. (2001) Differential regulation of gene expression in the obligate biotrophic interaction of Uromyces fabae with its host Vicia faba. Mol. Plant-Microbe Interact. 14, 1319-1326

34 Struck, C. et al. (2002) Characterization of a developmentally regulated amino acid transporter (AAT1p) of the rust fungus Uromyces fabae. Mol. Plant Pathol. 3, 23-30

35 Hahn, M. and Mendgen, K. (2001) Signal and nutrient exchange at biotrophic plant-fungus interfaces. Curr. Opin. Plant Biol. 4, 322-327
36 Struck, C. et al. (1998) The plasma membrane $\mathrm{H}^{+}$-ATPase from the biotrophic fungus Uromyces fabae: molecular characterisation of the gene (PMA1) and functional expression of the enzyme in yeast. Mol. Plant-Microbe Interact. $11,458-465$

37 Szabo, L.J. and Bushnell, W.R. (2001) Hidden robbers: the role of fungal haustoria in parasitism of plants. Proc. Natl. Acad. Sci. U. S. A. 98, 7654-7655

38 Heath, M.C. (1976) Ultrastructural and functional similarity of the haustorial neckband of rust fungi and the Casparian strip of vascular plants. Can. J. Bot. 54, 2484-2489

39 Murdoch, L.J. et al. (1998) Production and characterisation of monoclonal antibodies to cell wall components of the flax rust fungus. European J. Plant Pathol. 104, 331-346

40 Stark-Urnau, M. and Mendgen, K. (1995) Sequential deposition of plant glycoproteins and carbohydrates into the host-parasite interface of Uromyces vignae and Vigna sinensis. Evidence for endocytosis and secretion. Protoplasma 186,1-11

$41 \mathrm{Hu}$, G.G. and Rijkenberg, F.H.J. (1998) Subcellular localization of $\beta$-1,3-glucanase in Puccinia recondita f.sp. tritici-infected wheat leaves. Planta 204, 324-334

$42 \mathrm{Hu}$, G.G. and Rijkenberg, F.H.J. (1998) Ultrastructural localization of cytokinins in Puccinia recondita f.sp. tritici-infected wheat leaves. Physiol. Mol. Plant Pathol. 52, 79-94

43 Voegele, R.T. et al. (2001) Invertase in Uromyces fabae. Mol. Plant-Microbe Interact. University of Wisconsin, Madison, WI (ed.), Conference Proceedings, 445

44 Mims, C.W. et al. (2002) Ultrastructure of the host-pathogen interface in daylily leaves infected by the rust fungus Puccinia hemerocallidis. Protoplasma 219, 221-226

45 Moerschbacher, B.M. et al. (1999) Small oligomers of galacturonic acid are endogenous suppressors of disease resistance reactions in wheat leaves. J. Exp. Bot. 50, 605-612

46 Hall, J.L. and Williams, L.E. (2000) Assimilate transport and partitioning in fungal biotrophic interactions. Aust. J. Plant Physiol. $27,549-560$

47 Harrison, M.J. (1999) Biotrophic interfaces and nutrient transport in plant/fungal symbioses. J. Exp. Bot. 50, 1013-1022

48 Redman, R.S. et al. (2001) Fungal symbiosis from mutualism to parasitism: who controls the outcome, host or invader? New Phytol. $151,705-716$

49 Vogel, J. and Somerville, S. (2000) Isolation and characterization of powdery mildew-resistant Arabidopsis mutants. Proc. Natl. Acad. Sci. U. S. A. 97, 1897-1902

50 Cook, D. (1999) Medicago truncatula, a model in the making! Curr. Opin. Plant Biol. 2, 301-304

51 Heath, M.C. (2000) Advances in imaging the cell biology of plant-microbe interactions. Annu. Rev. Phytopathol. 38, 443-459

52 Howard, R.J. (2001) Cytology of fungal pathogens and plant-host interactions. Curr. Opin. Microbiol. 4, 365-373

53 Dumas, B. et al. (1999) Use of green fluorescent protein to detect expression of an endopolygalacturonase gene of Colletotrichum lindemuthianum during bean infection. Appl. Environ. Microbiol. 65, 1769-1771 